\title{
Comparing the Duration of Spinal Anesthesia Induced With Bupivacaine and a Bupivacaince-Lidocaine Combination in Trans-Urethral Resection of the Prostate (TURP)
}

\author{
Abdolhamid Chohedri ${ }^{1}$; Reza Raeesi Estabragh ${ }^{1, *}$; Mohammad Hossein Eghbal ${ }^{1}$; \\ Mohammad Ali Sahmeddini ${ }^{1}$; Hamidreza Eftekharian ${ }^{2}$; Ramita Shahabifar ${ }^{3}$ \\ ${ }_{2}^{1}$ Shiraz Anesthesiology and Critical Care Research Center, Namazi Hospital, Shiraz University of Medical Sciences, Shiraz, Iran \\ ${ }^{2}$ Department of Oral and Maxillofacial Surgery, Shahid Rajaei Hospital, Shiraz University of Medical Sciences, Shiraz, Iran \\ ${ }^{3}$ Student Research Committee, Namazi Hospital, Shiraz University of Medical Sciences, Shiraz, Iran \\ ${ }^{*}$ Corresponding author: Reza Raeesi Estabragh, Shiraz Anesthesiology and Critical Care Research Center, Namazi Hospital, Shiraz University of Medical Sciences, Shiraz, Iran. Tel: \\ +98-9376609727, Fax:+98-7136474270, E-mail: r.raeesi@yahoo.com
}

Received: November 28, 2014; Revised: April 4, 2015; Accepted: April 28, 2015

\begin{abstract}
Background: Spinal anesthesia is a safe anesthetic mode for transurethral prostate resection (TUPR). There are several studies assessing the effect of bupivacaine, lonely or accompanied by other drugs, on short duration operations. However, there is controversy regarding the exact combination.

Objectives:Theaim of the study was to compare the effects of spinal anesthesia with bupivacaine and low dose lidocaine with bupivacaine alone on postoperative pain in those undergoing transurethral resection of prostate(TURP).

Materials and Methods: This was a randomized clinical trial performed in Shiraz university of medical sciences during one year. Eighty men scheduled for TURP were randomly assigned to receive spinal anesthesia with $1.5 \mathrm{~mL}$ bupivacaine $0.6 \%$ and $0.6 \mathrm{~mL}$ Lidocaine $1 \%$ or spinal anesthesia with $1.5 \mathrm{~mL}$ bupivacaine $0.5 \%$ in combination with $0.6 \mathrm{~mL}$ normal saline. The primary endpoint was the time lag between induction of spinal anesthesia and reaching the highest spinal block level. We also recorded the duration of spinal block declining to L1 level, operation duration and the admission duration.

Results: Both study groups were comparable regarding the baseline characteristics. We did not find any difference between the two study groups regarding the duration of anesthetic block reaching the maximum level $(\mathrm{P}=0.433)$ and duration of decreasing it to $\mathrm{L} 1(\mathrm{P}=0.189)$. The course of postoperative recovery and duration of hospital admission were also comparable between the groups $(\mathrm{P}=0.661)$. Conclusions: Lidocaine does not have additive effects on duration and quality of spinal anesthesia with bupivacaine in those undergoing TURP.
\end{abstract}

Keywords: Spinal Anesthesia; Bupivacaine; Lidocaine

\section{Background}

Trans-urethral resection of the prostate (TURP) is one of the common surgical procedures $(1,2)$. It is still considered as the gold standard treatment of benign prostatic hyperplasia (BPH), which induces urinary obstruction and consequently, increases the risk of urinary tract infection. This kind of surgery is mostly performed for patients with prostate weight less than 60 grams (2). TURP, like other surgical procedures, may have several complications before and after surgery, which may cause some challenges for anesthetists. Some examples of them are TURP syndrome, bladder rupture and prostate capsule perforation (3). Early detection of such complications is depended on preserving the level of consciousness and brain function during the operation; thus, spinal anesthesia is preferred over general one in TURP (4).

Several studies compared the efficacy of spinal anesthesia versus general anesthesia in TURP. In a study performed by Rodgers (4), neuroaxial blockage had less mortality and morbidity compared to general anesthesia. Another study by Atashkhoii et al. (5) found that patients who underwent regional anesthesia have less post-operation pain compared to those undergoing general anesthesia. Moreover, Dobson et al. (6) declared that alterations in hemodynamic variables in TURP were greater by spinal anesthesia. On the other hand, it was seen that in elderly patients, severe and chronic hypotension can be occurred following spinal anesthesia, which may be due to sympathetic nerves blockage (7). Furthermore, bradycardia, urinary retention and neurological injuries can be mentioned as complications of spinal anesthesia, which is mostly dependent on the volume and dose of injection as well as the level of spinal block (4). Thus, to minimize such complications, especially in elderly patients with less compensatory capabilities, a

Copyright (C) 2015, Iranian Society of Regional Anesthesia and Pain Medicine(ISRAPM). This is an open-access article distributed under the terms of the Creative Commons Attribution-NonCommercial 4.0 International License (http://creativecommons.org/licenses/by-nc/4.0/) which permits copy and redistribute the material just in noncommercial usages, provided the original work is properly cited. 
high qualitative anesthesia with appropriate duration, dosing of drugs and minimal complications is needed to be introduced (4). Bupivacaine is a local long-acting anesthetic agent derived from amino-amides and used for reducing post-operational pain as well as peripheral nerve block (8). This drug, as a safe agent with minimal complications, is used for spinal anesthesia worldwide. Yet, because of its long acting effects, it is more preferable not to be used in short duration surgical procedures such as TURP (8-11).

There are several studies assessing the effect of bupivacaine, lonely or accompanied by other drugs, on short duration operations. Some declared that low dose hyperbaric bupivacaine in combination to Sufentanil can induce safe spinal anesthesia by reducing the risk of hypotension and the need of vasopressor drugs during the repair of hip fracture in elderly patients (12). Another survey conducted by Lee et al. (11) showed that using a mixture of Lidocaine and bupivacaine can shorten the duration of bupivacaine effects in spinal anesthesia and, thus, can provide rapid post-operational recovery.

Borazan et al. (13), revealed that using levobupivacaine compared to sufentanil can provide adequate spinal anesthesia without hemodynamic alterations in elderly patients. Another study suggested that bupivacaine-fentanyl combination can be a good choice for spinal anesthesia during TURP (8). On the other hand, Jacobsen et al. (10) showed that adding lidocaine to bupivacaine has no effect on the duration of spinal anesthesia. As it can be seen, there are some controversies on reducing the duration of spinal anesthesia by bupivacaine.

\section{Objectives}

Thus, in this study, we aimed to compare the duration of spinal anesthesia with bupivacaine versus bupivacaine in combination with lidocaine in patients undergoing TURP surgery.

\section{Materials and Methods}

This was a randomized clinical trial including patients scheduled for elective transurethral resection of the prostate (TURP) in Shahid Faghihi hospital, a tertiary healthcare center affiliated with Shiraz University of Medical Sciences during one year from August 2013 to August 2014. The study protocol was approved by both institutional review board (IRB) and medical ethics committee of Shiraz University of Medical Sciences. All patients provided their informed written consents before inclusion in the study. We included 80 men aged 50 to 70 years, ASA I and II with benign prostate hyperplasia (BPH) scheduled for TURP. Those patients with diabetes mellitus (DM), neurologic deficit and dysfunction, coagulopathy and lumbar spondylosis were excluded from the study. We also excluded those patients with infections over spine, spondylodiscitis, those with previous lumbar spine surgery, alcoholic patients and those who were opi- um and drug addicted. We also excluded those receiving glucocorticoids, immunosuppressives and analgesics before inclusion in the study.

All patients underwent complete history taking and clinical examination before inclusion in the study. Patients were given a sealed envelope containing the admission number. They were randomly assigned to two study groups using a computer-based random digit generator using the admission numbers given to the patients. The first group received $1.5 \mathrm{~mL}$ bupivacaine $0.5 \%$ (Bupivacaine-Merck®, Lyon, France) and $0.6 \mathrm{~mL}$ lidocaine 1\% (LIDOCAINE-EXIR@, Exir Pharmaceutical Company, Tehran, Iran) for induction of spinal anesthesia (Group 1); another group received $1.5 \mathrm{~mL}$ bupivacaine $0.5 \%$ in combination with $0.6 \mathrm{~mL}$ normal saline (Group 2).

In operation room, regular monitoring devices including electrocardiography, pulse-oximetry and sphygmomanometer were provided for each patient. Intravenous (i.v) access was secured with a 16 gauge cannula and $0.9 \%$ saline infused at a rate of $10 \mathrm{~mL} / \mathrm{kg}$ for preloading the circulation. Spinal anesthesia was induced in the sitting position under strict aseptic technique. The L3/L4 interspace was located and the skin was infiltrated with $2 \mathrm{~mL}$ of $2 \%$ lidocaine. A 25 gauge Quincke spinal needle was inserted at the L3/L4 inter-space and the space was confirmed by observation of free flow of cerebrospinal fluid. After injection of the prepared solutions, the patient was re-positioned supine with the head and shoulder supported with a pillow to prevent excessive rostral spread of the anesthetic, immediately. The level of sensory block was tested using the gentle pin-prick method and recorded. During the first 15 minutes, the sensory level was checked each minute. After that the sensory level was checked each 2.5 minutes. Sensory block level at T10 was considered as the point for initiating the operation. After the incision time, the sensory level was checked each 5 minutes until it declined to S2 level. Afterwards, it was checked each 10 minutes. The blood pressure was monitored every minute for 5 minutes and every 5 minutes until the end of procedure. Ephedrine was administered in a dosage of $4 \mathrm{mg} / \mathrm{kg}$ in case of decrease of blood pressure more than $20 \%$ or decline of systolic pressure to less than $100 \mathrm{~mm} / \mathrm{Hg}$. The time period between reaching the maximum point of sensory block and its reduction up to L1 was measured for each patient. Afterwards, the patients were transferred to recovery rooms if they had stable vital signs, complete consciousness, no active hemorrhage, no severe pain and S2 or lower sensory block level. The primary endpoint was the time lag between induction of spinal anesthesia and reaching the highest spinal block level. We also recorded the duration of spinal block decline to L1 level, operation duration and the admission duration.

All statistical analyses were performed using statistical package for social sciences (SPSS Inc., Chicago, USA) version 16.0. Data are presented as mean \pm SD and proportions as appropriate. Parametric variables were com- 
pared between the two study groups using independent sample t-test, while chi-square test was used to compare the proportions. A 2-sided P-value of less than 0.05 was considered statistically significant.

\section{Results}

Overall, we found 86 patients eligible for the study of whom six were excluded due to not tolerating the spinal anesthesia and receiving general anesthesia. Thus, each group included 40 patients. All patients finished the study and thus the final number of patients in the final analysis was 80 (Figure 1). Table 1 summarized baseline characteristics of 80 patients undergoing TURP with spinal anesthesia in the two study groups. There was no significant difference between the two study groups regarding the baseline characteristics. The duration of operation was comparable between the two study groups $(\mathrm{P}=0.074)$.

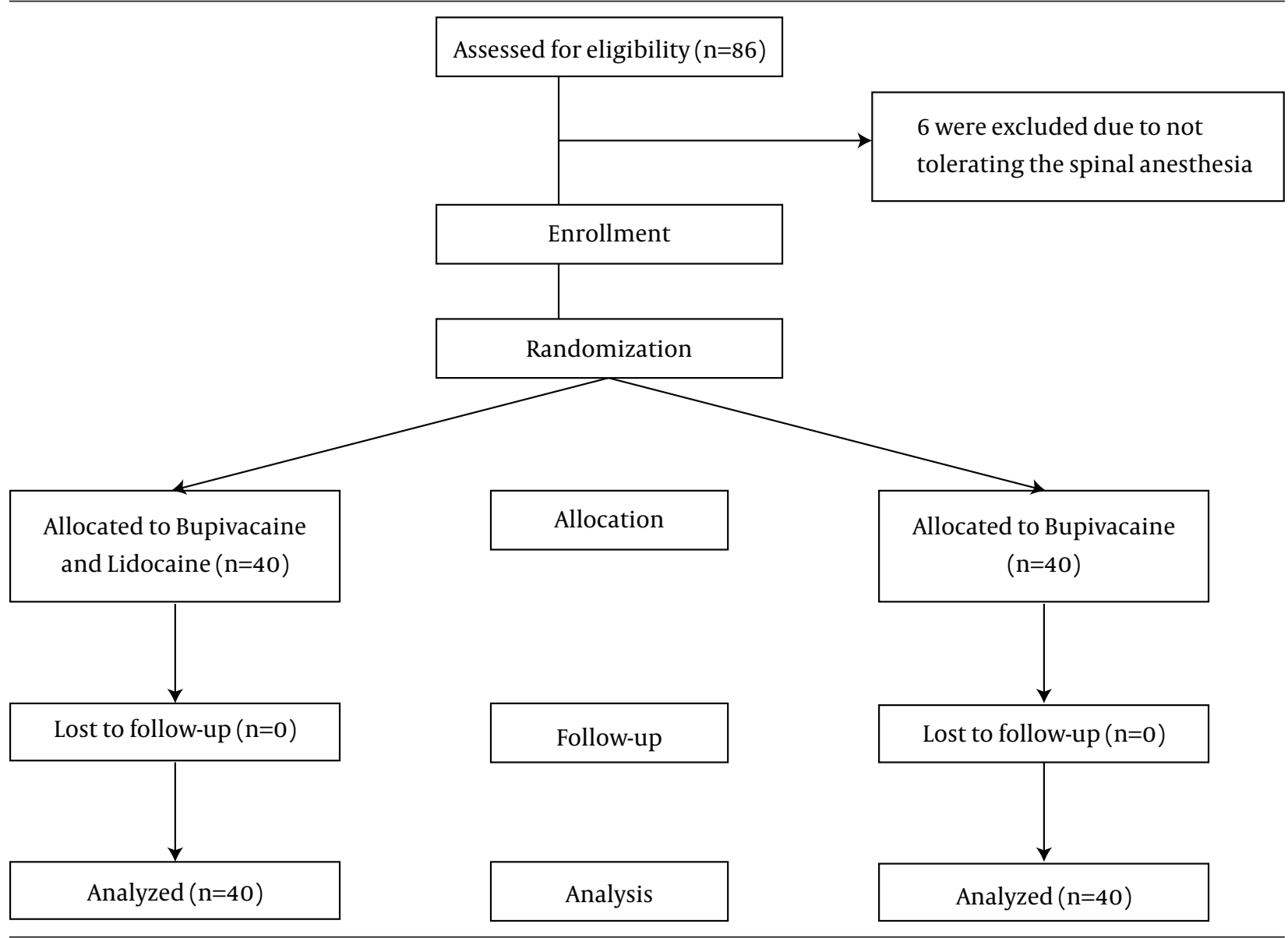

Figure 1. CONSORT Flow Diagram of the Study

Table 1. Baseline Characteristics of 80 Patients Undergoing TURP With Spinal Anesthesia Using Two Different Protocol ${ }^{\text {a }}$

\begin{tabular}{|c|c|c|c|}
\hline Variables & $\operatorname{Group} 1(n=40)$ & Group $2(n=40)$ & P Value \\
\hline Age, $y$ & $65.9 \pm 6.9$ & $64.1 \pm 8.7$ & 0.362 \\
\hline Height, cm & $172 \pm 7.4$ & $168 \pm 16.1$ & 0.189 \\
\hline Weight, kg & $69.2 \pm 10.2$ & $70.5 \pm 11.9$ & 0.661 \\
\hline BMI, $\mathbf{k g} / \mathbf{m}^{2}$ & $23.4 \pm 2.8$ & $25.4 \pm 5.4$ & 0.139 \\
\hline Operation duration, min & $47.2 \pm 18.2$ & $49.1 \pm 13.3$ & 0.739 \\
\hline ASA & & & 0.699 \\
\hline $\mathrm{I}$ & $12(30)$ & $14(35)$ & \\
\hline II & $28(70)$ & $26(65)$ & \\
\hline
\end{tabular}

\footnotetext{
a Data are presented as No. $(\%)$ or mean \pm SD.
} 
Chohedri A et al.

Table 2. The Outcome of Spinal Anesthesia Using Bupivacaine and Lidocaine (Group 1) or Bupivacaine Alone (Group 2) in 80 Patients Undergoing TURP

\begin{tabular}{lccc}
\hline Variables & Group 1 $(\mathbf{N}=\mathbf{4 0})$ & Group 2 $(\mathbf{N}=\mathbf{4 0})$ & P Value \\
\hline $\begin{array}{l}\text { Lag Time for Reaching the Maximum Level of } \\
\text { Spinal Block, min }\end{array}$ & $7.3 \pm 1.9$ & $6.8 \pm 2.1$ & 0.433 \\
Duration of Decreasing Block Level to L1, min & $74.1 \pm 10.4$ & $69.5 \pm 10.1$ & 0.189 \\
Duration of Admission to Recovery Room, $\mathbf{h}$ & $67.3 \pm 19.2$ & $65.6 \pm 10.3$ & 0.661 \\
Maximum block level & $\mathrm{T} 8$ & $\mathrm{~T} 8$ & 0.999 \\
\hline
\end{tabular}

The characteristics of the operation and the study outcome are demonstrated in Table 2 . We found that the duration of reaching to maximum sensory block level was not significantly different between the two study groups. In the same way, the duration of decreasing sensory level to L1 was comparable between the two study groups. Both groups had comparable postoperative hospitalization course in recovery rooms. We also found that the maximum sensory level was not different between the two study groups (Table 2 ).

\section{Discussion}

Currently, spinal anesthesia is considered more efficient and safer compared with general anesthesia for many surgical procedures. TURP as one of the most common urologic surgeries can be performed by spinal anesthesia. Although several studies have investigated the best combination of drugs used in spinal anesthesia during TURP $(8,14-16)$, the issues remain controversial. In this study, we tried to compare the quality of spinal anesthesia with combination of bupivacaine and lidocaine to bupivacaine alone in patients undergoing TURP. We found that the two protocols were not different regarding quality and duration of spinal anesthesia. Thus adding lidocaine to bupivacaine is not advantageous for improving the quality of spinal anesthesia in those undergoing TURP.

In a systematic review, Rodgers et al. reviewed 141 clinical trials with 9559 patients; they found that the mortality rate was decreased about a third in patients who received spinal anesthesia. Details revealed that spinal anesthesia can reduce deep vein thrombosis by $44 \%$, pulmonary embolism by $55 \%$, transfusion requirements by $50 \%$, pneumonia by $39 \%$ and respiratory depression by $59 \%$ (4). In another trial, 22 patients who underwent TURP were divided into two groups. One received general anesthesia, while the other group received spinal one. It was seen that general anesthesia can cause reduction in cardiac output, mean arterial pressure and heart rate; however, spinal block only decreased the mean arterial pressure (6).

The most common anesthetic agent recently became popular globally is bupivacaine. Anesthesiologists are interested in this agent due to its safety and minimal disadvantages. Yet, due to its long acting effect, it is not ap- propriate for short acting operations $(7,11,17)$. However, several studies were conducted to assay bupivacaine's efficacy in short acting surgeries. Ben-David et al. (9), compared different doses of bupivacaine in spinal anesthesia for arthroscopy. They used bupivacaine in doses of 5, 7.5, 10 and $15 \mathrm{mg}$. All 4 doses were then diluted by normal saline and prescribed in a volume of $3 \mathrm{~mL}$. They found that $7.5 \mathrm{mg}$ bupivacaine of $0.5 \%$ bupivacaine in $8 \%$ dextrose diluted with an equal volume of saline was the most acceptable dose for ambulatory arthroscopy; moreover, this dose had the most appropriate recovery profile for this surgery (10). In another trial conducted in 2004 by Olofsson et al. (7), 50 elderly patients intended to undergo hip fracture repair, were divided into two groups. One received hyperbaric bupivacaine $7.5 \mathrm{mg}$ and sufentanil $5 \mu \mathrm{g}$, while the other group received $15 \mathrm{mg}$ bupivacaine. Comparing hemodynamic instability of the two groups revealed that combination of bupivacaine and sufentanil provided more reliable spinal anesthesia with less hemodynamic changes such as hypertension and need for vasopressor drugs (7). Lee et al. (11), declared that using $6 \mathrm{mg}$ lidocaine in combination with $7.5 \mathrm{mg}$ bupivacaine can shorten the duration of bupivacaine-induced anesthesia, thus, may result in more rapid post-operational recovery. This study was performed on 90 patients divided into three groups. $1.5 \mathrm{mg}$ bupivacaine $5 \%$, used intrathecally, added to saline (group 1), lidocaine 1\% (group 2) and lidocaine $2 \%$ (group 3 ) (11).

In 2009, a study conducted on 70 patients scheduled for TURP found that using low dose of bupivacaine mixed with Sufentanil or fentanyl can induce sufficient and effective spinal anesthesia with no hemodynamic instability or long-term post-surgical recovery in elderly patients. They also reported that Sufentanil was superior to fentanyl (7). Another study indicated that inducing spinal anesthesia in knee arthroscopy, using lidocaine plus bupivacaine compared to bupivacaine alone, had no effect on the duration of anesthesia (5). Another trial introduced the combination of bupivacaine and fentanyl as a good choice for TURP. In this trial, 60 patients undergone TURP surgery were divided into two groups. One received bupivacaine mixed with fentanyl versus a group which received prilocaine combined with fentanyl. They declared that bupivacaine and fentanyl can provide more sufficient anesthesia with more stable hemodynamic profiles as well as shorter block duration (9). 
In conclusion, adding lidocaine to bupivacaine for spinal anesthesia in patients undergoing TURP does not improve the quality and duration of anesthesia. Lidocaine does not have additive effects on duration and quality of spinal anesthesia with bupivacaine in those undergoing TURP. Further clinical trials are required to shed light on the issue.

\section{Acknowledgements}

The author would like to acknowledge all patients and their families who patiently participated in the current study. We would also like to acknowledge the assistance of Dr. Fariborz Ghaffarpasand in scientific and technical editing of the manuscript.

\section{Authors' Contributions}

Abdolhamid Chohedri contributed in study concept and design, data collection, critical revision and approval of the manuscript. Reza Raeesi Estabragh, Mohammad Hossein Eghbal, Mohammad Ali Sahmeddini, Hamidreza Eftekharian and Ramita Shahabifar contributed in study concept and design, data collection and approval of the manuscript.

\section{Funding/Support}

The study was supported by a grant from Shiraz University of Medical Sciences, Shiraz, Iran.

\section{References}

1. Malhotra V. Transurethral Resection of the Prostate. Anesthesiol Clin North Am. 2000;18(4):883-97.

2. Cornu J, Ahyai S, Bachmann A, De la Rosette J, Gilling P, Gratzke $\mathrm{C}$, et al. A systematic review and meta-analysis of functional outcomes and complications following transurethral procedures for lower urinary tract symptoms resulting from benign prostatic obstruction: an update. European Urol. 2015;67(6):1066-96.

3. Kim NY, Kim SY, Ju HM, Kil HK. Selective spinal anesthesia using $1 \mathrm{mg}$ of bupivacaine with opioid in elderly patients for transurethral resection of prostate. Yonsei Med J. 2015;56(2):535-42.

4. Rodgers A. Reduction of postoperative mortality and morbid- ity with epidural or spinal anaesthesia: results from overview of randomised. Bmj. 2000;321(7275):1493.

5. Atashkhoii S, Jafari Shobeiri M, Azarfarin R. Intraperitoneal and incisional bupivacaine analgesia for major abdominal/gynecologic surgery: A placebo-controled trial. Vol. 20. National Research Centre of Medical Sciences of I.R. IRAN; 2008.

6. Dobson PM, Caldicott LD, Gerrish SP, Cole JR, Channer KS. Changes in haemodynamic variables during transurethral resection of the prostate: comparison of general and spinal anaesthesia. $\mathrm{Br} J$ Anaesth.1994;72(3):267-71.

7. Olofsson C, Nygards EB, Bjersten AB, Hessling A. Low-dose bupivacaine with sufentanil prevents hypotension after spinal anesthesia for hip repair in elderly patients. Acta Anaesthesiol Scand. 2004;48(10):1240-4.

8. Akcaboy ZN, Akcaboy EY, Mutlu NM, Serger N, Aksu C, Gogus N. Spinal Anesthesia with Low-Dose Bupivacaine-Fentanyl Combination: A Good Alternative for Day Case Transurethral Resection of Prostrate Surgery in Geriatric Patients. Brazil JAnesthesiol. 2012;62(6):753-61.

9. Ben-David B, Levin H, Solomon E, Admoni H, Vaida S. Spinal bupivacaine in ambulatory surgery: the effect of saline dilution. Anesth Analg. 1996;83(4):716-20.

10. Jacobsen J, Husum B, von Staffeldt H, Qvist TF, Jensen PE, Kledal $\mathrm{S}$. The addition of lidocaine to bupivacaine does not shorten the duration of spinal anesthesia: a randomized, double-blinded study of patients undergoing knee arthroscopy. Anesth Analg. 2011;113(5):1272-5.

11. Lee SJ, Bai SJ, Lee JS, Kim WO, Shin YS, Lee KY. The duration of intrathecal bupivacaine mixed with lidocaine. Anesth Analg. 2008;107(3):824-7.

12. Le Guen H, Roy D, Branger B, Ecoffey C. Comparison of fentanyl and sufentanil in combination with bupivacaine for patientcontrolled epidural analgesia during labor. J Clin Anesth. 2001;13(2):98-102

13. Borazan H, Davarcı I, Kececioglu A, Otelcioglu S. The effects of low dose levobupivacaine with or without sufentanil intrathecally in transurethral resection of prostate. Eur J Gen Med. 2011;8(2):134-40.

14. Kararmaz A, Kaya S, Turhanoglu S, Ozyilmaz MA. Low-dose bupivacaine-fentanyl spinal anaesthesia for transurethral prostatectomy. Anaesthesia. 2003;58(6):526-30.

15. Sirivanasandha B, Lennox PH, Vaghadia H. Transurethral resection of the prostate (TURP) with low dose spinal anesthesia in outpatients: a 5 year review. Can J Urol. 2011;18(3):5705-9.

16. Zohar E, Noga Y, Rislick U, Leibovitch I, Fredman B. Intrathecal anesthesia for elderly patients undergoing short transurethral procedures: a dose-finding study. Anesth Analg. 2007;104(3):552-4.

17. Gupta S, Sampley S, Kathuria S, Katyal S. Intrathecal sufentanil or fentanyl as adjuvants to low dose bupivacaine in endoscopic urological procedures. J Anaesthesiol Clin Pharmacol. 2013;29(4):509-15. 